\title{
Tai Chi and Qigong Practices for Chronic Heart Failure: A Systematic Review and Meta-Analysis of Randomized Controlled Trials
}

\author{
Xiankun Chen $\left(\mathbb{D},{ }^{1,2,3}\right.$ Gianluigi Savarese, ${ }^{4}$ Yiyi Cai, ${ }^{3,5}$ Liuling Ma, ${ }^{3,5}$ Cecilia Stålsby Lundborg, \\ Wei Jiang, ${ }^{3,5}$ Zehuai Wen $(1),{ }^{2,6}$ Weihui Lu $(1), 3,5$ and Gaetano Marrone ${ }^{1}$ \\ ${ }^{1}$ Department of Global Public Health, Health Systems and Policy, Karolinska Institute, 17177 Stockholm, Sweden \\ ${ }^{2}$ Key Unit of Methodology in Clinical Research, Guangdong Provincial Hospital of Chinese Medicine, 510120 Guangzhou, China \\ ${ }^{3}$ The Second Affiliated Hospital of Guangzhou University of Chinese Medicine, 510405 Guangzhou, China \\ ${ }^{4}$ Department of Medicine, Cardiology, Karolinska Institutet, 17176 Stockholm, Sweden \\ ${ }^{5}$ Department of Cardiology, Guangdong Provincial Hospital of Chinese Medicine, 510120 Guangzhou, China \\ ${ }^{6}$ National Centre for Design Measurement and Evaluation in Clinical Research, Guangzhou University of Chinese Medicine, \\ 510405 Guangzhou, China \\ ${ }^{7}$ Heart Failure Center/Department of Cardiology, Guangdong Provincial Hospital of Chinese Medicine, \\ 510120 Guangzhou, China
}

Correspondence should be addressed to Zehuai Wen; wenzh@gzucm.edu.cn and Weihui Lu; weihui.lu@163.com

Received 26 July 2020; Revised 28 November 2020; Accepted 1 December 2020; Published 15 December 2020

Academic Editor: Liye Zou

Copyright $\odot 2020$ Xiankun Chen et al. This is an open access article distributed under the Creative Commons Attribution License, which permits unrestricted use, distribution, and reproduction in any medium, provided the original work is properly cited.

\begin{abstract}
Background. Several randomized controlled trials (RCTs) have assessed the role of Tai Chi and Qigong Practices (TQPs) in managing chronic heart failure (CHF). They have included broad variations in comparators, sample sizes, and results. This study evaluates existing RCTs for evidence of TQPs rehabilitation effects for CHF. Methods. Both English and Chinese databases were searched from their inception to October 23, 2019. RCTs were included if they compared the addition of TQPs into routine managements (RMs) to RMs alone or compared TQPs to general exercise, with RMs as a consistent cointervention in both groups. Data were screened and extracted independently using predesigned forms. RCT quality was assessed with the Cochrane tool. The primary outcomes were peak oxygen consumption $\left(\mathrm{VO}_{2 \text { peak }}\right), 6$-minute walking distance (6MWD), and Minnesota Living with Heart Failure Questionnaire (MLHFQ). Mean differences (MDs) and 95\% confidence intervals (CIs) were calculated, and heterogeneity was assessed with an $I^{2}$ statistic. Results. A total of 33 RCTs with 2,465 patients were included in the systematic review. Compared to the RMs alone, TQPs plus RMs improved $\mathrm{VO}_{2 \text { peak }}$ (MD: $1.24 \mathrm{~mL} / \mathrm{kg} / \mathrm{min}, 95 \% \mathrm{CI}, 0.91$ to $1.57 ; I^{2}=0 \%$ ), 6MWD (MD: 59.63 meters, $95 \%$ CI, 43.35 to $75.90 I^{2}=88 \%$ ), and MLHFQ (MD: -8.63 scores; $95 \%$ CI, -10.60 to $-6.67 ; I^{2}=94 \%$ ). Compared to general exercise, superior improvements were found in the TQP group; they were significant in MLHFQ (MD: -9.18 scores; $95 \%$ CI, -17.95 to $-0.41 ; I^{2}=86 \%$ ), but not in $\mathrm{VO}_{2 \text { peak }}$ or $6 \mathrm{MWD}$. Evidence was also found of TQPs' safety and high adherence. Conclusions. Considering that there are low costs, multiple physical benefits, and no equipment required, TQPs are a promising rehabilitation therapy, as an adjunct to routine pharmacotherapies or as an alternative to conventional exercises, especially in home-based settings.
\end{abstract}

\section{Introduction}

Heart failure is a global pandemic affecting at least 26 million people worldwide, and its prevalence is increasing [1]. Although pharmacological therapy is the primary treatment, exercise-based cardiac rehabilitation (EBCR) has become an important recommendation in clinical guidelines. Unfortunately, few patients with chronic heart failure (CHF) participate in EBCR, due to the lack of resources [2]. Those patients who do participate in supervised cardiac 
rehabilitation programs show low adherence [3]. Therefore, finding a cheap, convenient form of EBCR with high compliance is of growing importance [4].

Qigong is an adaptable form of exercise that can be practiced at any place, any time, without any specialized equipment, and with minimal time investment. Hence, it is easily incorporated into daily routines and could be integrated into a comprehensive cardiac rehabilitation program. Qigong is an ancient Chinese martial art; it is an umbrella term, covering a spectrum of exercises including Dao-YinShu (physical and breathing exercises), Wu-Qin-Xi (five animals play), Baduanjin (eight silken movements), and YiJin-Jing (changing tendons exercises) [5]. Tai Chi is a wellknown exercise that has grown from the Qigong tradition. Tai Chi and Qigong Practices (TQPs) typically involve slow movements synchronized with meditation and regulated breathing techniques [5]. Moreover, all TQPs share the principle that any form of Qigong has an effect on the cultivation of balance and the harmony of vital energy (qi), which functions as a holistic, coherent, and interactive system [5].

In recent years, the rehabilitating effects of TQPs for CHF patients have received increasing recognition and attention. An earlier systematic review of TQPs in cardiac rehabilitation, including seven randomized or nonrandomized controlled trials, suggested that TQPs enhance physical health and promote overall quality of life among patients with chronic heart disease [6]. This has been corroborated by a recent systematic review which included 35 randomized controlled trials (RCTs) with 2,249 patients to evaluate TQPs' effect on cardiovascular diseases [7]. However, these two reviews included studies without subgroup analysis by varying cardiovascular diseases. Therefore, the rehabilitating effects of TQPs for heart failure remain unclear.

In light of the growing number of RCTs of TQPs used for rehabilitation in $\mathrm{CHF}$ patients, and the ensuing need for critical evaluation, we conducted a systematic review and meta-analysis of the available evidence to inform clinical practice. The research questions were as follows: (1) Does the addition of TQPs into routine managements (RMs) improve clinical outcomes, as compared to RMs alone? (2) What is the difference between TQPs and general exercise in terms of improving CHF patients' clinical outcomes? (3) What is TQPs' safety profile and how is adherence?

\section{Methods}

This systematic review was conducted and reported in accordance with the Preferred Reporting Items for Systematic Reviews and Meta-Analyses statement (Supplementary Materials: PRISMA 2009 Checklist) [8] and the Cochrane Handbook for Interventional Reviews [9]. The study protocol has been published previously in PROSPERO (CRD42018081982).

2.1. Search Strategy. The electronic databases PubMed, Cochrane Central Register of Controlled Trials (CENTRAL), EMBASE, CINAHL, and China National Knowledge
Infrastructure were searched from their inceptions until October 23, 2019. Searches were restricted to English and Chinese. Search strategy details are provided in the Supplemental Materials-Search strategy.

2.2. Study Selection. One reviewer (XC) scanned all titles and abstracts to exclude irrelevant citations which were checked by a second reviewer (YC). Two reviewers (XC and $\mathrm{YC}$ ) independently assessed the eligibility of the remaining citations after retrieving the full texts of potentially relevant articles. Randomized controlled trials (RCTs) were included, and selection criteria conformed to the PICOS approach, as described hereinafter.

2.2.1. Populations. Patients diagnosed with heart failure that were in a stable phase of the disease with no acute exacerbations were included. There were no restrictions regarding heart failure subtypes.

2.2.2. Intervention. RCTs that applied an intervention group receiving any form of Qigong practice (e.g., Tai Chi, Liu-ZiJue, Baduanjin, Wu-Qin-Xi, and Yi-Jin-Jing) were included. However, studies were excluded if TQPs had been used in combination with other oral traditional interventions such as Chinese herbal decoctions.

2.2.3. Comparators. No exercise or general exercise is planned, structured, and repetitive for the purpose of conditioning the body (such as walking, cycling, swimming, or running). RMs were provided according to clinical guidelines and as a consistent cointervention to both groups.

2.2.4. Outcomes. Primary outcomes included (1) peak oxygen consumption $\left(\mathrm{VO}_{2 \text { peak }}\right),(2) 6$-minute walking distance (6MWD), and (3) disease-specific quality of life using total scores according to the Minnesota Living with Heart Failure Questionnaire (MLHFQ). Secondary outcomes included left ventricular ejection fraction (LVEF), B-type natriuretic peptide (BNP), and other clinically relevant outcomes, as well as adverse events and participant's adherence to TQPs.

2.3. Data Extraction. The data extraction form was drafted based on the reporting guidelines from the extended CONSORT statement for randomized trials of nonpharmacologic treatment [10] and the TIDieR (Template for Intervention Description and Replication) [11]. One reviewer (XC) used a standardized form to extract data from the included articles. The following data were extracted: study characteristics (e.g., author, year, and country), participant characteristics such as age, sex, sample size, New York Heart Association (NYHA) functional class, LVEF subtype, TQPs interventions (e.g., TQP types, frequency, durations, and target intensity), and controls, as well as outcomes measured.

Attempts were made to contact the original investigators regarding any missing data. The extracted data was checked by a second reviewer (YC). Any discrepancies were resolved 
by agreement after rechecking the source papers and further discussion with a third reviewer (LM).

2.4. Quality and Certainty of Evidence. In accordance with recommendations in the Cochrane Handbook, the trials' methodological quality was independently evaluated by two reviewers (XC and $\mathrm{YC}$ ) using the Cochrane risk-of-bias assessment tool. Any discrepancies were resolved by agreement after rechecking the source papers and further discussion with a third reviewer (LM). The following domains were considered: (1) random sequence generation, (2) allocation concealment, (3) blinding of patients and personnel, (4) blinding of outcome assessors for primary outcomes, (5) incomplete outcome data, and (6) selective reporting. The overall evidence and certainty of evidence were evaluated with the Grading of Recommendations Assessment, Development, and Evaluation approach.

2.5. Data Analysis. RevMan 5.3 (Cochrane Collaboration) and STATA 12 (StataCorp) were used to analyze the data from the included studies. The outcome measures from the individual trials were combined through meta-analysis, when possible. For each outcome measure, studies were pooled separately according to comparators: (A) TQPs plus RMs vs. RMs: to evaluate add-on effects of TQPs; and (B) TQPs plus RMs vs. general exercises plus RMs: to contrast TQPs and general exercise. For studies with multiple control groups $[12,13]$, such as TQPs plus RMs vs. general exercise plus RMs control vs. RMs control, the results were split into pairwise comparisons by the different comparators. For the study which had two TQP groups using the same exercise but with different practice times (30 minutes or 60 minutes) [14], the results of these two TQPs groups were combined and used as the mean effects of the TQP group.

Given that all variables in the included studies consisted of continuous data, we used the mean difference (MD) when the same instrument was used, or the standardized mean difference (SMD) when different instruments were used, with $95 \%$ confidence intervals (CIs) to analyze the outcomes. A $p$ value $<0.05$ was considered statistically significant. For trials that had missing information for outcome means or standard deviations (SD), data was first sought from the original investigators. If it was not available from the author, then imputations were performed using the recommended statistical approaches mentioned in the Cochrane Handbook (session 6.5.2.3). For those studies using the median and interquartile range (IQR) instead of means and $\mathrm{SD}$, the medians were used as a substitute for the means, and the SD was approximated as $\mathrm{SD}=\mathrm{IQR} / 1.35$. For those studies with only missing SD, SD was imputed from a reported standard error, CI, $t$-statistic, $Z$-statistic, or $p$ value that was correlated with the difference between the two groups' means.

Heterogeneity was assessed with a chi-square test ( $p<0.10$ was considered indicative of statistical significance) and an $I^{2}$ statistic (where $I^{2}>30 \%, 50 \%$, and $75 \%$ indicated moderate, substantial, or considerable heterogeneity, respectively) [9]. A random-effects model was employed where there was formal evidence of statistical heterogeneity
$\left(I^{2}\right.$ statistic $\left.>50 \%\right)$. Otherwise, a fixed-effects model was used. Potential publication bias was evaluated by visual examination of funnel plot asymmetry and Egger's test ( $p$ value $<0.05$ was considered statistically significant). When the number of articles included in one analysis was limited (i.e., less than 10), publication bias was not assessed.

A sensitivity analysis was conducted by removing each study individually to estimate the results' consistency. When there was heterogeneity, subgroup analyses were performed to identify its potential sources. Subgroup analyses were performed according to different types of TQPs used in the program (Tai Chi, Qigong, Tai Chi plus Qigong) and program duration (in weeks). Additionally, a post hoc subgroup analysis was conducted to explore heterogeneity originating from the heart failure subtype (HFrEF or HFpEF).

\section{Results}

In total, 1,480 records were retrieved from database searches. After excluding duplicates, 999 potentially relevant abstracts were screened, and 934 were excluded for failing to meet the inclusion criteria. The remaining 65 full texts were read, and 33 RCTs [12-44] (24 in Chinese $[12,14,18-22$, 24-30, 34-42, 44] and 9 in English [13, 15-17, 23, 31-33, 43]) were deemed eligible for this systematic review. The quantitative synthesis was performed with 32 RCTs by pooling the results through a meta-analysis. A flowchart of the study selection process is shown in eFigure 1 in Supplementary Materials.

\subsection{Characteristics of Included Studies}

3.1.1. Study Characteristics. The characteristics of the included studies are shown in Table 1. The studies were published between 2004 and 2019. States or regions of publication were China $(n=25)[12,14,17-22,24-30$, $34-42,44]$, Taiwan $(n=2)[17,44]$, the United States $(n=5)$ $[13,23,31-33]$, the United Kingdom $(n=1)$ [15], Italy $(n=1)$ [16], and Sweden $(n=1)$ [43].

3.1.2. Participants. The included RCTs involved a total of 2,465 CHF patients (age ranging from 52 to $74 \mathrm{yrs)} \mathrm{with}$ NYHA functional class ranging from I to IV. The sample size per RCT ranged from 16 to $180,84 \%$ of whom were Chinese.

3.1.3. Intervention. For the TQP exercise program type, researchers used Tai Chi in 17 RCTs $[13,14,16,18,19$, 22-25, 30-33, 35, 38, 41, 43], Qigong in 14 RCTs, and Tai Chi plus Qigong in the remaining two RCTs [15, 40]. Qigong included Baduanjin [17, 21, 26, 27, 36, 37, 39, 42], Liuzijue [12], Baduanjin plus Liuziju [20, 28, 29, 34], and ChanChuang [44]. TQP exercise time lasted from 15 to 60 minutes per session, and TQP program duration varied between 4 weeks $(n=2)[19,36], 8$ weeks $(n=1)[21], 12$ weeks $(n=17)[12,16,17,23-27,31-33,35,37-40,44], 16$ weeks $(n=3)[13,15,43], 24$ weeks $(n=5)[14,18,22,30,41]$, and 52 weeks $(n=5)[20,28,29,34,42]$. 


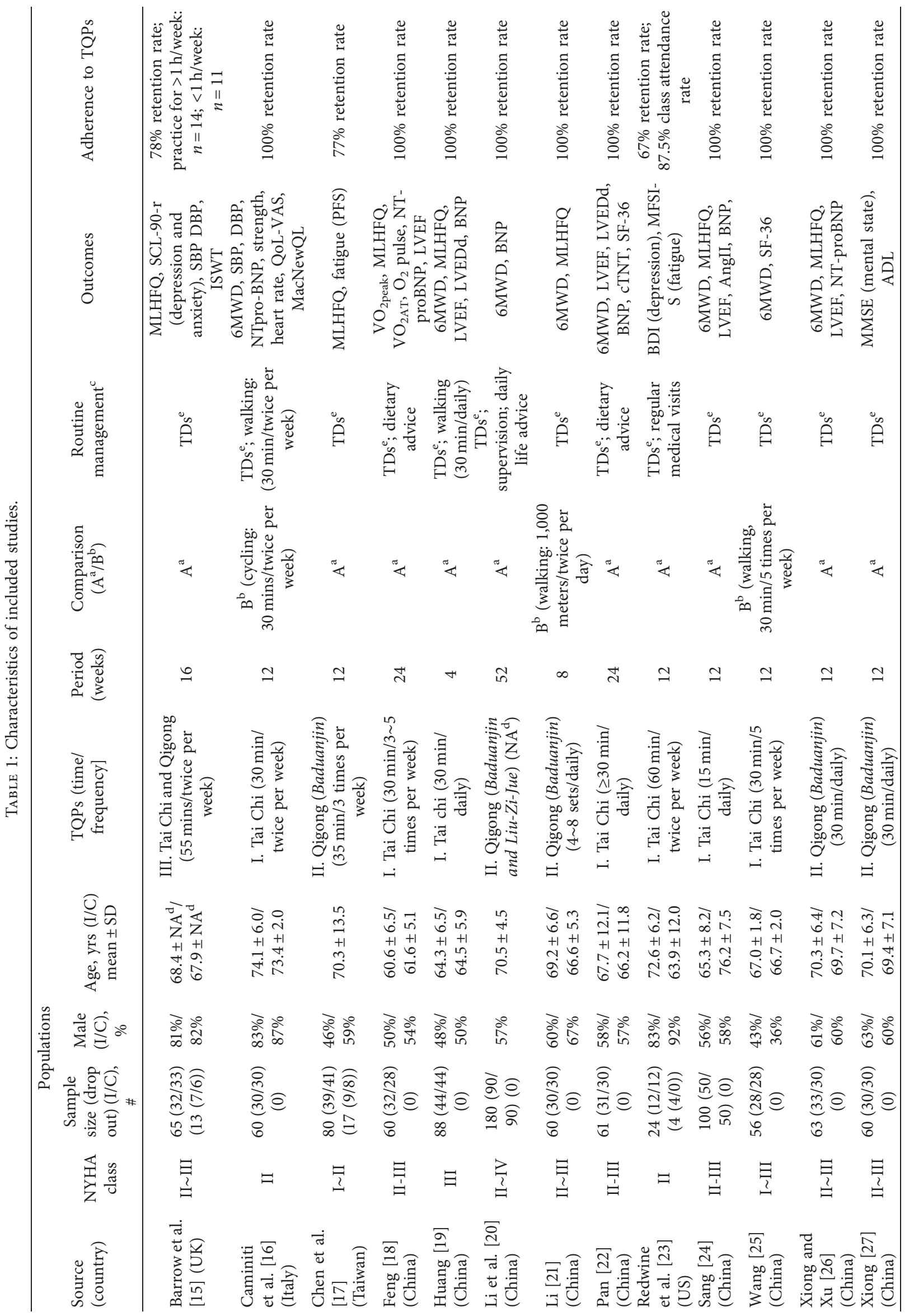




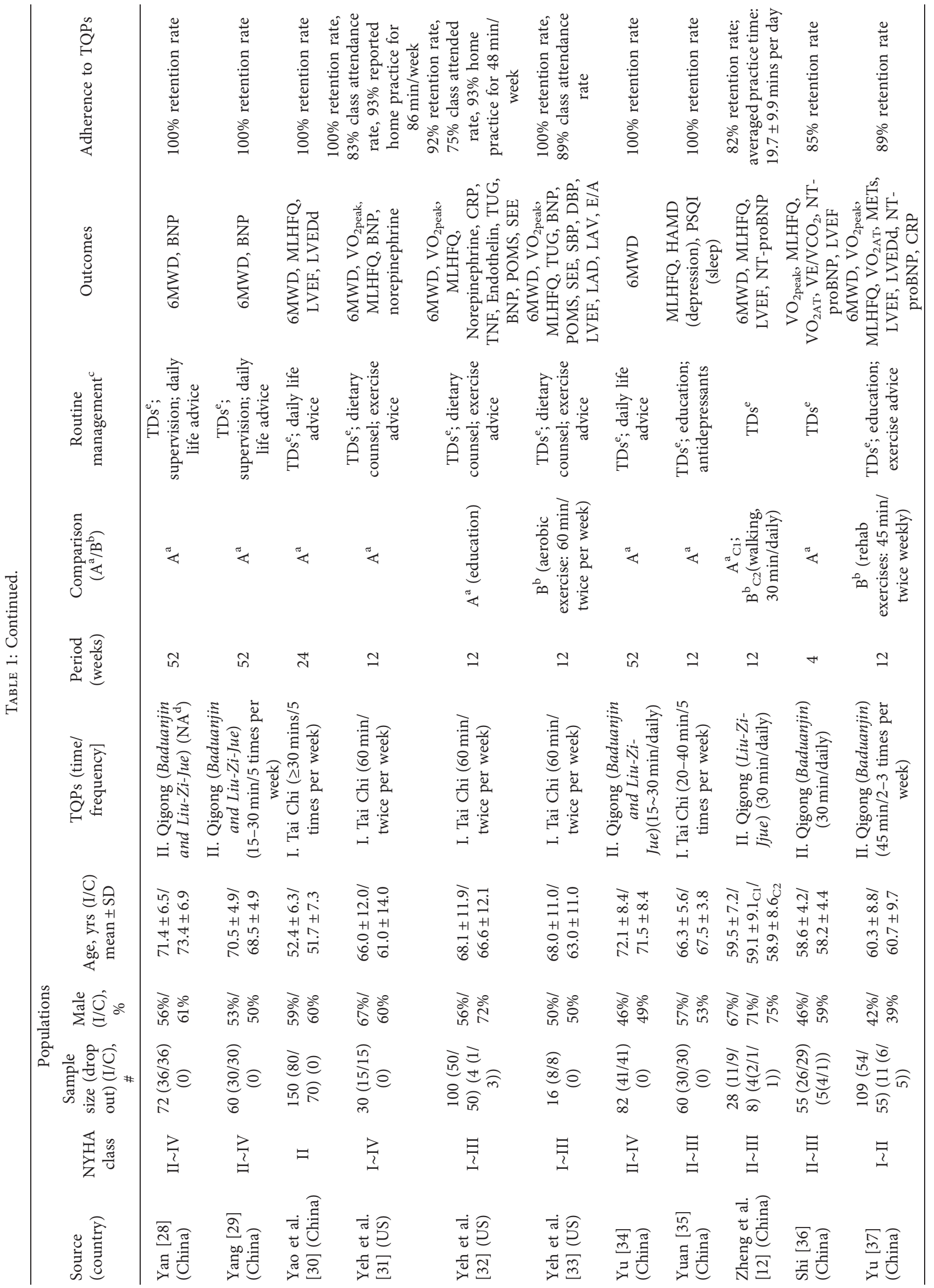




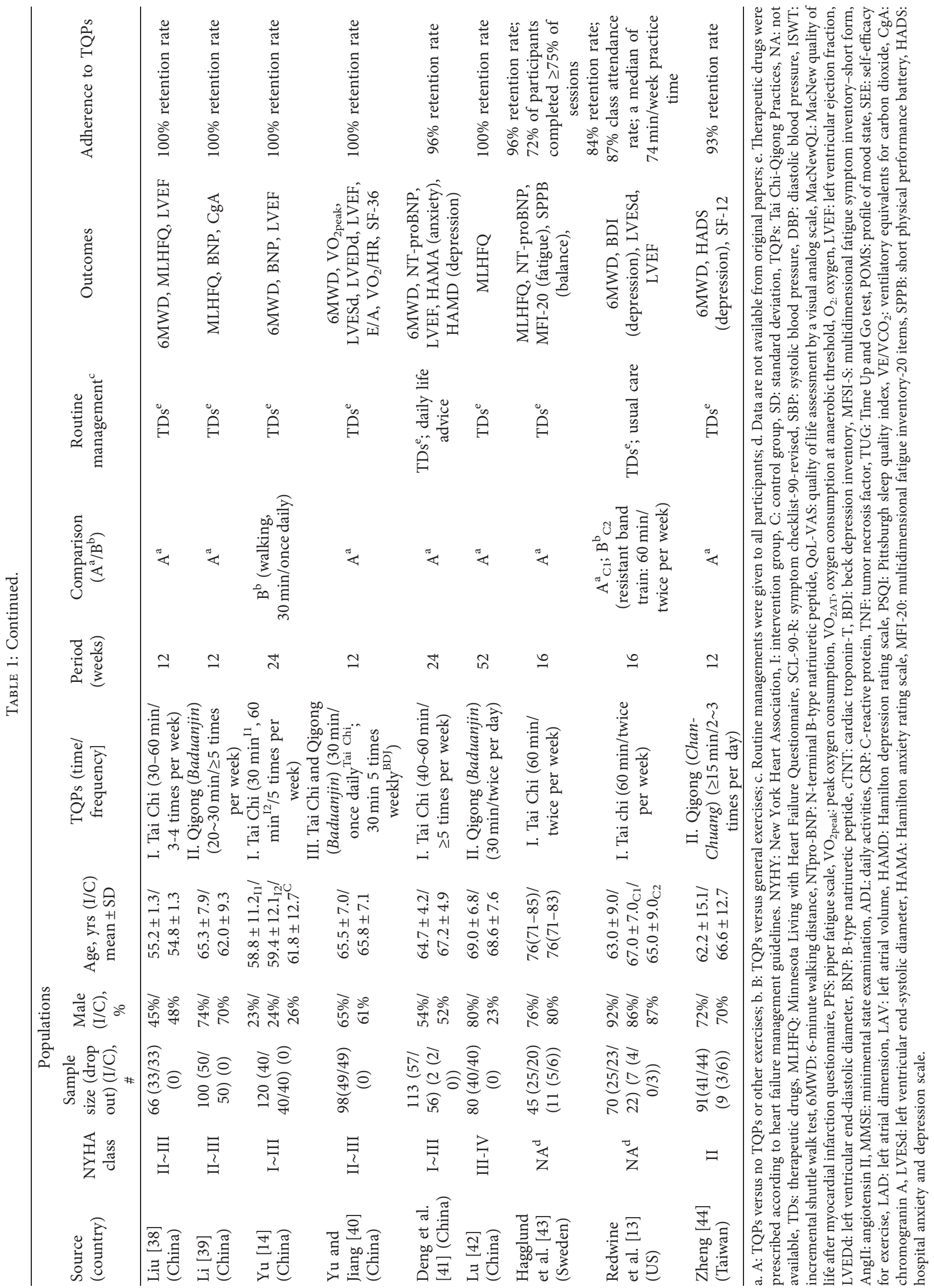


3.1.4. Comparators. In terms of the comparison, 26 studies compared TQPs plus RMs against RMs alone [15-20, 22-24, 26-32, 34-36, 38-44]. Five studies compared TQPs plus RMs against general exercises plus RMs $[14,21,25,33,37]$, one of which applied two TQP groups using Tai Chi with different practice times ( 30 minutes vs. 60 minutes) [14]. There were two 3 -arm studies that included two control groups comparing TQPs plus RMs vs. general exercises plus RMs vs. RMs [12,13]. Generally, RMs included standard pharmacological treatments in all of the RCTs; in some of the RCTs, it also included education, dietary counseling, and/or general exercise advice.

3.1.5. Outcomes. Results of three primary outcomes (MLHFQ, 6MWD, and $\mathrm{VO}_{2 \text { peak }}$ ) and two secondary outcomes (LVEF and BNP) are summarized in Figure 1.

3.2. MLHFQ. (A) TQPs plus RMs versus RMs. The addition of TQPs produced a statistically significant lower MLHFQ score (better quality of life), but the heterogeneity was considerable (14 RCT $[12,15,17-19,24,26,30-32,35,36$, $38,42], n=10,000$; MD: -8.63 scores; $95 \% \mathrm{CI},-10.60$ to $-6.67 ; I^{2}=94 \%$; Figure 2A). The subgroup analysis based on TQP types and program durations did not resolve the heterogeneity (Figure $2 \mathrm{~A}$ ).

(B) TQPs versus General Exercise. The pooled results also showed statistically significant lower MLHFQ scores in the TQPs group than in the general exercise group, but again heterogeneity was considerable (4 RCTs $[12,21,33,37], n=203$; MD: -9.18 scores; $95 \%$ CI, -17.95 to $-0.41 ; I^{2}=86 \%$; Figure $2 B$ ). Subgroup analyses revealed that TQP durations and HF subtype might explain the heterogeneity (Figure 2B).

\section{3. $6 M W D$}

3.3.1. TQPs plus RMs versus RMs. The pooled results from 17 RCTs [12, 13, 19, 20, 22, 24, 26, 28-32, 34, 38, 40, 41, 44] $(n=1,416)$ showed that adding TQPs led to a statistically significant 56.52 meter increase in 6MWD (95\% CI, 41.27 to $71.78)$, but the heterogeneity was considerable $\left(I^{2}=88 \%\right.$; Figure $3 \mathrm{~A}$ ). In the subgroup analyses, the heterogeneity declined in the Qigong subgroup and the 12-week subgroup (Figure 3A).

3.3.2. TQPs versus General Exercise. The pooled results, though not statistically significant, showed that TQPs were more effective, but with considerable heterogeneity (7 RCTs [12-14, 16, 21, 33, 37], $n=428$; MD: 46.66 meters; 95\% CI, -18.17 to $111.49 ; I^{2}=98 \%$ ) (Figure 3B). Deleting the study with the stronger effect [21] reduced the heterogeneity to $I^{2}=20 \%$, without changing the results' significance (Supplementary Materials: eTable 1). TQP durations might also explain the heterogeneity (Figure 3B).
3.4. $\mathrm{VO}_{2 \text { peak }}$

3.4.1. TQPs plus RMs versus $R M$ s. The pooled results from 4 RCTs $[18,31,32,36,37](n=245)$ showed that adding TQPs into RMs can lead to a statistically significant $1.24 \mathrm{~mL} / \mathrm{kg} /$ min improvement in patients' $\mathrm{VO}_{2 \text { peak }}(95 \% \mathrm{CI}, 0.91$ to 1.57 ; $I^{2}=0 \%$; Figure $4 \mathrm{~A}$ ).

3.4.2. TQPs versus General Exercise. The pooled MD, although not statistically significant, showed that TQPs were more effective (2 RCTs [33, 37], $n=125 ; \mathrm{MD}: 0.14 \mathrm{~mL} / \mathrm{kg} /$ $\min , 95 \% \mathrm{CI},-0.43$ to $0.7 ; I^{2}=0 \%$; Figure $\left.4 \mathrm{~B}\right)$. Both of these RCTs included HFpEF patients, and both lasted for 12 weeks.

3.5. Secondary Outcomes. The addition of TQPs showed a small but significant improvement in the LVEF (Figure 5A), as well as in the BNP (Figure 6A). Similarly, when compared to general exercises, the pooled MD showed that TQPs were more effective for the LVEF (Figure 5B), as well as for the BNP (Figure 6B). However, neither was statistically significant. Results of other secondary clinical outcomes reported in more than one study are presented in Table 2.

3.6. Sensitivity Analysis. Sensitivity analysis showed that most of the pooled results were robust when removing each study individually. When comparing TQPs with general exercises, the beneficial effects of TQPs on MLHFQ became statistically insignificant if 2 studies were removed individually (Supplementary Materials: eTable 1). For the pool of 6MWD comparing TQPs with general exercises, deleting one study [21] made the beneficial effects of TQPs significant and reduced the heterogeneity from $I^{2}=97 \%$ to $I^{2}=20 \%$ (Supplementary Materials: eTable 1). In the BNP sensitivity analysis, deleting Pan's study [22] reduced the heterogeneity from $I^{2}=95 \%$ to $I^{2}=39 \%$, without changing the results' significance (Supplementary Materials: eTable 1).

3.7. TQP Safety and Patient Adherence. No adverse event related to TQPs was found in the included studies, and patient dropout in the TQP groups was low, with most withdrawals being due to hospitalization or CHF exacerbation. TQP adherence was good. The retention rate in the TQP groups ranged from $67 \%$ to approximately $100 \%$. The six studies that included TQP training classes reported attendance between $75 \%$ and $89 \%[13,23,31-33,43]$ (Table 1).

3.8. Evidence Quality and Certainty. Individual items on the risk-of-bias assessment are shown in Figures 12 and 13 in Supplementary Materials. As TQP is an exercise training, designing an experiment with a credible placebo-control arm is challenging. Thus, all RCTs were open-label. All studies were unclear on outcome assessment blinding except for two RCTs $[32,33]$ in which the authors claimed that the outcome assessors had been blind to patient treatment allocation. Only half of the RCTs provided adequate random sequence generation $[16-19,22,25,29,35-40,44]$, and only 


\begin{tabular}{|c|c|c|c|c|c|c|c|c|c|c|c|c|c|}
\hline \multirow[b]{2}{*}{$\begin{array}{l}\text { Outcomes } \\
\text { (measurements) }\end{array}$} & \multicolumn{4}{|c|}{ Source } & \multicolumn{3}{|c|}{ Effect size } & \multirow[b]{2}{*}{$\begin{array}{l}\text { Level of } \\
\text { Certainty }\end{array}$} & \multicolumn{5}{|c|}{ Certainty assessment } \\
\hline & $\begin{array}{l}\text { Countries } \\
\text { (year range) }\end{array}$ & NYHA & LVEF range & $\begin{array}{l}\text { No. of } \\
\text { patients } \\
\text { (RCTs) }\end{array}$ & $\begin{array}{l}\text { Mean difference } \\
\quad(95 \% \mathrm{CI})\end{array}$ & $I^{2}$ & $\begin{array}{l}\text { Referred } \\
\text { figure }\end{array}$ & & Risk of bias & Inconsistency & Indirectness & Imprecision & $\begin{array}{l}\text { Publication } \\
\text { bias }\end{array}$ \\
\hline \multicolumn{14}{|c|}{ A. $\lceil$ Taichi-Qigong-Practice plus $R M\rfloor v \varsigma\lceil R M\rfloor$} \\
\hline $\begin{array}{l}\text { - Qualigy of life } \\
\text { (MLHFQ) } \\
\text { FU: 4 52 wks }\end{array}$ & $\begin{array}{l}\text { China/Taiwan/ } \\
\text { US/UK } \\
\text { (2004 2019) }\end{array}$ & I IV & $\begin{array}{c}22 \sim 60 \%(n=687) \\
\leq 50 \%(n=115) \\
\text { unknown }(n=198)\end{array}$ & $\begin{array}{l}1000 \\
(14)\end{array}$ & $\begin{array}{c}-8.63 \text { scores } \\
(-10.60 \text { to }-6.67)\end{array}$ & $94 \%$ & Figure 2A & Moderate & Not serious & Serious $^{c}$ & Not serious & Not serious & Not serious \\
\hline $\begin{array}{l}\text { - Exercise capacity } \\
\text { (6MWD) } \\
\text { FU: } 4 \sim 52 \mathrm{wks}\end{array}$ & $\begin{array}{l}\text { China/Taiwan/ } \\
\text { US } \\
(2004 \sim 2019)\end{array}$ & $\mathrm{I} \sim \mathrm{IV}$ & $\begin{array}{c}22 \sim 52 \%(n=865) \\
\text { unknown }(n=551)\end{array}$ & $\begin{array}{l}1416 \\
(17)\end{array}$ & $\begin{array}{c}59.63 \text { meters } \\
(43.35 \text { to } 75.90)\end{array}$ & $88 \%$ & Figure 3A & Moderate & Not serious & Serious $^{c}$ & Not serious & Not serious & Not serious \\
\hline $\begin{array}{l}\text { - Exercise function } \\
\left(\text { Peak } \mathrm{VO}_{2}\right) \\
\text { FU: } 4 \sim 24 \text { wks }\end{array}$ & $\begin{array}{l}\text { China/US } \\
\text { (2004 2018) }\end{array}$ & I IV & $\begin{array}{c}22 \sim 42 \%(n=190) \\
\leq 50 \%(n=55)\end{array}$ & $\begin{array}{l}245 \\
(4)\end{array}$ & $\begin{array}{l}1.24 \mathrm{~mL} / \mathrm{kg} / \mathrm{min} \\
(0.91 \text { to } 1.57)\end{array}$ & $0 \%$ & Figure $4 \mathrm{~A}$ & Moderate & Not serious & Not serious & Not serious & Serious $^{\mathrm{d}}$ & Undetected \\
\hline $\begin{array}{l}\text { - Echocardiography } \\
\text { (LVEF) } \\
\text { FU: } 4 \sim 24 \text { wks }\end{array}$ & $\begin{array}{c}\text { China } \\
(2010 \sim 2019)\end{array}$ & I $\sim$ III & $\begin{array}{c}30 \sim 52 \% \leq(n=747) \\
50 \%(n=55) \\
\text { unknown }(n=66)\end{array}$ & $\begin{array}{l}868 \\
(11)\end{array}$ & $\begin{array}{c}3.97 \% \\
\text { (1.22 to } 6.72)\end{array}$ & $96 \%$ & Figure 5A & Moderate & Not serious & Serious $^{c}$ & Not serious & Not serious & Not serious \\
\hline $\begin{array}{l}\text { - Laboratory test } \\
\text { (BNP) } \\
\text { FU: } 4 \sim 52 \mathrm{wks}\end{array}$ & $\begin{array}{l}\text { China/US } \\
(2004 \sim 2019)\end{array}$ & $\mathrm{I} \sim \mathrm{IV}$ & $\begin{array}{c}22 \sim 49 \%(n=279) \\
\geq 50 \%(n=100) \\
\text { unknown }(n=312)\end{array}$ & $\begin{array}{l}691 \\
(8)\end{array}$ & $\begin{array}{c}-76.12 \mathrm{pg} / \mathrm{mL} \\
(-134.61 \text { to }-17.62)\end{array}$ & $95 \%$ & Figure 6A & Moderate & Not serious & Serious $^{c}$ & Not serious & Not serious & Undetected \\
\hline \multicolumn{14}{|c|}{ B. $\lceil$ Taichi-Qigong-Practice plus $R M\rfloor v$ s $\lceil$ General Exercises plus $R M\rfloor$} \\
\hline $\begin{array}{l}\text { - Qualigy of life } \\
\text { (MLHFQ) } \\
\text { FU: 8 12 wks }\end{array}$ & $\begin{array}{l}\text { China/US } \\
\text { (2013 2018) }\end{array}$ & I III & $\begin{array}{c}52 \sim 65 \%(n=34) \\
\geq 40 \%(n=109) \\
\text { unknown }(n=60)\end{array}$ & $\begin{array}{l}203 \\
(4)\end{array}$ & $\begin{array}{c}-9.18 \text { scores } \\
(-17.95 \text { to }-0.41)\end{array}$ & $86 \%$ & Figure 2B & Low & Serious $^{\mathrm{a}}$ & Serious $^{c}$ & Not serious & Serious $^{\mathrm{d}}$ & Undetected \\
\hline $\begin{array}{l}\text { - Exercise capacity } \\
\text { (6MWD) } \\
\text { FU: } 12 \sim 24 \text { wks }\end{array}$ & $\begin{array}{l}\text { China/ } \\
\text { Italy/US } \\
(2011 \sim 2019)\end{array}$ & I $\sim \mathrm{III}$ & $\begin{array}{c}31 \sim 65 \%(n=260) \\
\geq 40 \%(n=108) \\
\text { unknown }(n=60)\end{array}$ & $\begin{array}{l}428 \\
(7)\end{array}$ & $\begin{array}{c}46.66 \text { meters } \\
(-18.17 \text { to } 111.49)\end{array}$ & $97 \%$ & Figure 3B & Low & Serious ${ }^{b}$ & Serious $^{c}$ & Not serious & Serious $^{\mathrm{d}}$ & Undetected \\
\hline $\begin{array}{l}\text { - Exercise function } \\
\text { (Peak VO2) } \\
\text { FU: } 12 \mathrm{wks}\end{array}$ & $\begin{array}{l}\text { China/US } \\
\text { (2013 2018) }\end{array}$ & I III & $\begin{array}{l}62 \sim 65 \%(n=16) \\
\geq 40 \%(n=109)\end{array}$ & $\begin{array}{l}125 \\
(2)\end{array}$ & $\begin{array}{l}0.14 \mathrm{~mL} / \mathrm{kg} / \mathrm{min} \\
(-0.43 \text { to } 0.70)\end{array}$ & $0 \%$ & Figure $4 \mathrm{~B}$ & Moderate & Not serious & Not serious & Not serious & Serious $^{\mathrm{d}}$ & Undetected \\
\hline $\begin{array}{l}\text { - Echocardiography } \\
\text { (LVEF) } \\
\text { FU: } 12 \sim 24 \mathrm{wks}\end{array}$ & $\begin{array}{l}\text { China/US } \\
(2013 \sim 2019)\end{array}$ & $\mathrm{I} \sim \mathrm{III}$ & $\begin{array}{c}31 \sim 65 \%(n=273) \\
\geq 40 \%(n=109)\end{array}$ & $\begin{array}{l}382 \\
(4)\end{array}$ & $\begin{array}{c}3.17 \% \\
(-1.25 \text { to } 7.59)\end{array}$ & $0 \%$ & Figure 5B & Moderate & Not serious & Not serious & Not serious & Serious $^{\mathrm{d}}$ & Undetected \\
\hline $\begin{array}{l}\text { - Laboratory test } \\
\text { (BNP) } \\
\text { FU: } 12 \sim 24 \mathrm{wks}\end{array}$ & $\begin{array}{l}\text { China/US } \\
\text { (2013 2019) }\end{array}$ & $\mathrm{I} \sim \mathrm{III}$ & $31 \sim 65 \%(n=136)$ & $\begin{array}{l}136 \\
(2)\end{array}$ & $\begin{array}{c}61.29 \mathrm{pg} / \mathrm{mL} \\
(-52.22 \text { to } 174.79)\end{array}$ & $77 \%$ & Figure 6B & Low & Not serious & Serious $^{c}$ & Not serious & Serious ${ }^{\mathrm{d}}$ & Undetected \\
\hline
\end{tabular}

FIGURE 1: Summary of findings. a: 30\% of the information from 1 high-risk RCT; removing this RCT significantly altered the effect estimates; $25 \%$ of the studies had a high risk of the randomization process. b: $20 \%$ of the information from 1 high-risk RCT; removing this RCT moderately altered the size of effect estimates; $17 \%$ of the studies had a high risk of the randomization process. c: considerable heterogeneity based on $I^{2}$. d: imprecision, as OIS criteria were not met, primarily due to the small sample size (<400). 6MWD: 6-minute walking distance, BNP: B-type natriuretic peptide, CI: confidence interval, FU: follow-up, LVEF: left ventricular ejection fraction, MLHFQ: Minnesota Living with Heart Failure Questionnaire, NYHA: New York Heart Association, Peak VO2: peak oxygen consumption, RCT: randomized controlled trial, RM: routine management (according to current guidelines).

five RCTs reported allocation concealment methods $[13,17,31,37,44]$. Twenty-nine $(88 \%)$ studies were at unclear risk of selective reporting because neither protocol nor trial registration info was available.

Publication bias analyzed by funnel plots showed only minor asymmetry (Supplementary Materials: eFigure 14), and there was no evidence of funnel plot asymmetry for 6MWD $(p=0.10)$, MLHFQ $(p=0.817)$, or LVEF $(p=0.929)$. Thus, a publication bias mechanism is not a major cause for concern.

Evidence from RCTs indicated (with a moderate level of certainty) that the addition of TQPs into RMs was associated with a better quality of life, improved exercise capacity, increased LVEF, and reduced BNP level, as compared with the associated with the RMs alone. Low evidence certainty showed that TQPs were associated with a larger improvement in the quality of life and exercise capacity than general exercise (Figure 1).

\section{Discussion}

This systematic review identified 33 RCTs with a total of 2,465 participants to evaluate the evidence of rehabilitative effects from TQPs for patients with CHF. There are three main findings. (1) When compared to RMs, TQPs plus RMs improved $\mathrm{VO}_{2 \text { peak }}, 6 \mathrm{MWD}$, and MLHFQ, and the pooled effects were robust with heterogeneity found in 6MWD and MLHFQ, but not in $\mathrm{VO}_{2 \text { peak }}$. In addition, TQPs might have beneficial effects on other clinical outcomes such as LVEF and BNP. (2) When compared to general exercise, superior improvements were found in the TQP group; they were significant in MLHFQ, but not in $\mathrm{VO}_{2 \text { peak }}, 6 \mathrm{MWD}, \mathrm{LVEF}$, or BNP. (3) Evidence was also found that TQP is safe and that there is high adherence to TQP programs.

Quality of life and exercise capacity are two different domains of interest in rehabilitation research [45]. The positive results of MLHFQ suggest that TQPs can improve CHF patients' quality of life. The present findings are consistent with those of other systematic reviews and metaanalyses of RCTs of Tai Chi for CHF [46-48]. Moreover, the pooled effects are also clinically significant, with the effect estimate being greater than the minimally important clinical difference of 5 points [49] (Figure 2). TQPs are characterized by the interplay between flowing circular physical postures and movements, mindful awareness, and breathing techniques in a harmonious manner [5]. Hence, they exert less 


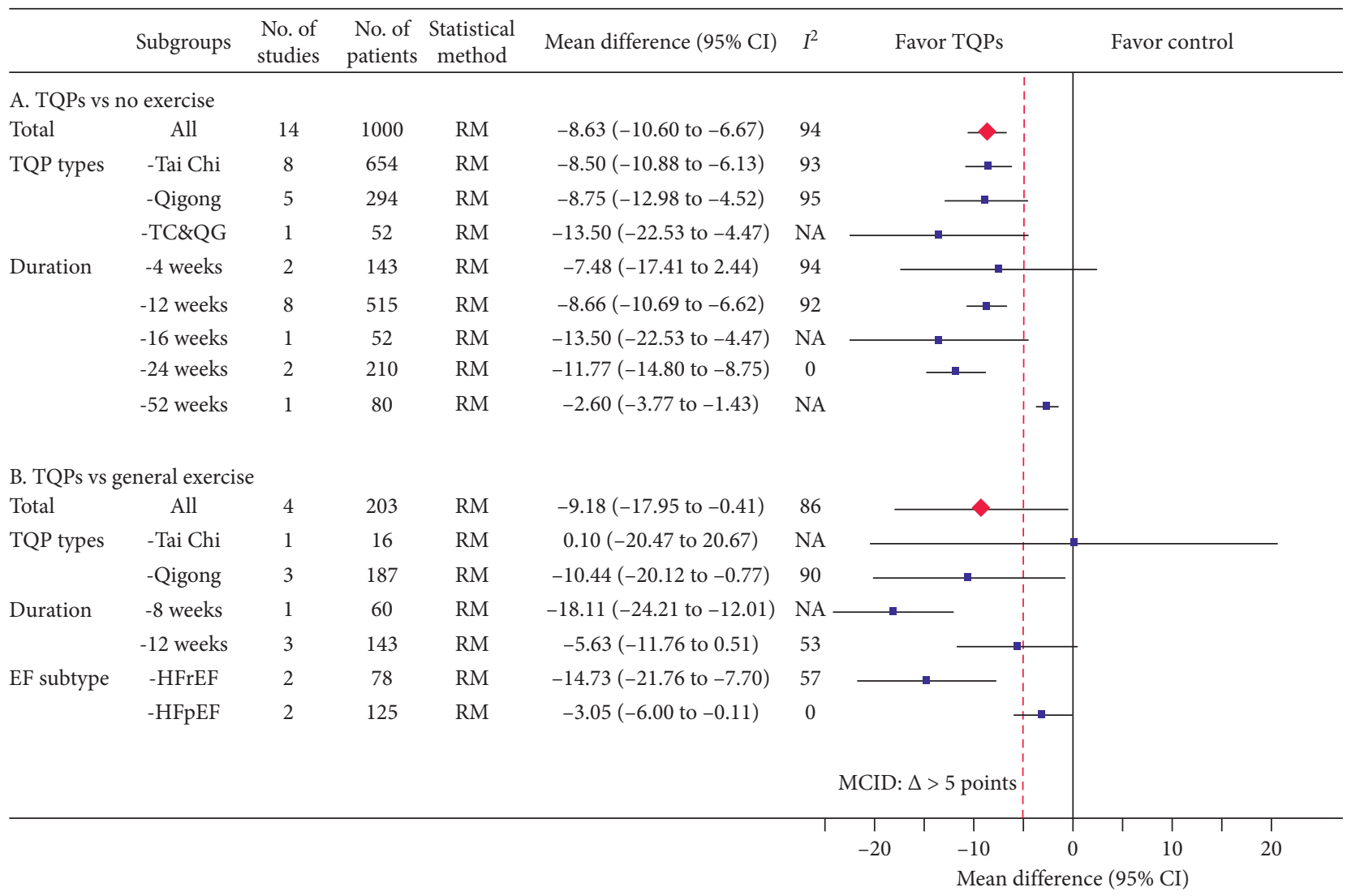

FIgURE 2: Meta-analysis results for MLHFQ (total score) including overall pooled effects and subgroup effects. The pooled effect from all included studies and each subgroup is shown in red diamond and blue square, respectively. Both are along with their 95\% confidence intervals. Meta-analysis results showing individual study data are presented in Supplementary Materials: eFigures 2 and 3 for part A and part B, respectively. MLHFQ: Minnesota Living with Heart Failure Questionnaire, No.: number, CI: confidence interval, TQP: Tai Chi and Qigong Practice, NA: not available, TC\&QG: Tai Chi and Qigong, RM: random-effects model, MCID: minimum clinically important difference, EF: ejection fraction, HFrEF: heart failure with reduced ejection fraction, HFpEF: heart failure with perceived ejection fraction.

cardiopulmonary stress and enable the body to relax after practicing. They also address breathing, a crucial aspect of $\mathrm{CHF}$ management. Slowing breathing patterns allows more complete inspiration/expiration and gas exchange in patients with CHF [50]. This mitigates their symptoms, thus improving their quality of life.

We found a moderate level of evidence that the addition of TQPs into RMs benefits CHF patients' exercise capacity. Firstly, positive results were found for the $6 \mathrm{MWD}$, both in the overall pooling of 17 studies (60 meters) and in each of the TQP subgroups. Our results are consistent with a previous systematic review of Tai Chi which reported a similar improvement of 50 meters [46]. The pooled improvements were also clinically relevant because they are larger than the minimum clinically important difference ( $>30$ meters) [51].

Secondly, positive results were also found for the $\mathrm{VO}_{2 \text { peak }}$ which is the gold standard for assessing exercise capacity [52]. This reinforces the beneficial gains in exercise capacity by practicing TQPs for CHF patients. Unlike findings from previous reviews which reported that Tai Chi could not change the $\mathrm{VO}_{2 \text { peak }}$ [46-48], there was a positive result for $\mathrm{VO}_{2 \text { peak }}$ in the Tai Chi subgroup in our study. Our meta-analysis involves two more recent RCTs $[18,36]$ which were not included in those previous meta-analyses. This results in a larger sample size. In addition, we have restricted our attention to those studies without exercise controls. Studies with exercise controls, similar to the active-control in the pharmacological trials, are expected to have negative results and the effects' estimates are usually smaller than those of placebo-control trials, especially for the open-label studies.

Evidence from the RCTs indicated (with a low level of certainty) that TQPs may have rehabilitation effects on quality of life and exercise capacity that are similar to those of general aerobic exercises. Our findings show that TQPs' benefits are superior to those of general exercise at improving quality of life (MD: -9.18). This has not been reported in earlier studies. The effect size is similar to that reported in a Cochrane systematic review [53], in which the reviewers compared all exercise interventions with usual care $(-7.11$ points, $95 \% \mathrm{CI}-10.49$ to -3.73$)$. In addition, the magnitude of the pooled improvements from the TQPs in $\mathrm{VO}_{2 \text { peak }}$ and 6MWD was similar to that of the pooled improvements from conventional exercise modalities in other systematic reviews, respectively $[54,55]$. Moreover, although the pooled effect estimate for 6MWD was not statistically 


\begin{tabular}{|c|c|c|c|c|c|c|c|c|}
\hline & Subgroups & $\begin{array}{l}\text { No. of } \\
\text { studies }\end{array}$ & $\begin{array}{c}\text { No. of } \\
\text { patients }\end{array}$ & $\begin{array}{c}\text { Statistical } \\
\text { method }\end{array}$ & Mean difference $(95 \% \mathrm{CI})$ & $I^{2}$ & Favor control & Favor TQPs \\
\hline \multicolumn{9}{|c|}{ A. TQPs vs no exercise } \\
\hline Total & All & 17 & 1416 & RM & $59.63(43.35$ to 75.9$)$ & 88 & & - \\
\hline \multirow{3}{*}{ TQP types } & -Tai Chi & 9 & 754 & $\mathrm{RM}$ & 50.99 (27.01 to 74.97$)$ & 92 & & $1=$ \\
\hline & -Qigong & 7 & 564 & $\mathrm{RM}$ & 74.11 (59.50 to 88.71$)$ & 0 & & $\rightarrow$ \\
\hline & -TC\&QG & 1 & 98 & RM & 49.47 (33.95 to 64.99$)$ & NA & & - \\
\hline \multirow[t]{5}{*}{ Duration } & -4 weeks & 1 & 88 & $\mathrm{RM}$ & $0.53(-13.08$ to 14.14$)$ & NA & & \\
\hline & -12 weeks & 8 & 564 & RM & 48.95 (34.32 to 63.59$)$ & 59 & & - \\
\hline & -16 weeks & 1 & 48 & $\mathrm{RM}$ & $-9.00(68.69$ to 50.69$)$ & NA & $\longrightarrow$ & - \\
\hline & -24 weeks & 3 & 322 & RM & 88.32 (34.00 to 142.65$)$ & 94 & & - \\
\hline & -52 weeks & 4 & 394 & $\mathrm{RM}$ & $81.42(60.66$ to 102.18$)$ & 0 & & $\rightarrow$ \\
\hline \multicolumn{9}{|c|}{ B. TQPs vs general exercise } \\
\hline Total & All & 7 & 428 & RM & $46.66(-18.17$ to 111.49$)$ & 97 & & \\
\hline \multirow[t]{2}{*}{ TQP types } & -Tai Chi & 4 & 243 & RM & $14.35(-10.11$ to 38.81$)$ & 32 & & \\
\hline & -Qigong & 3 & 185 & $\mathrm{RM}$ & $93.10(-33.13$ to 219.34$)$ & 99 & & \\
\hline \multirow[t]{5}{*}{ Duration } & -8 weeks & 1 & 55 & RM & 181.89 (162.26 to 201.52$)$ & NA & & $\rightarrow$ \\
\hline & -12 weeks & 4 & 223 & $\mathrm{RM}$ & 22.65 (12.09 to 33.21$)$ & 0 & & \\
\hline & -16 weeks & 1 & 62 & RM & $-51.00(-122.85$ to 10.85$)$ & NA & 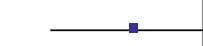 & \\
\hline & -24 weeks & 1 & 120 & $\mathrm{RM}$ & $26.35(-3.67$ to 56.37$)$ & NA & & \\
\hline & & & & & & & & MCID: $\Delta>30$ meters \\
\hline
\end{tabular}

Figure 3: Meta-analysis results for 6MWD (meters) including overall pooled effects and subgroup effects. The pooled effect from all included studies and each subgroup is shown in red diamond and blue square, respectively. Both are along with their 95\% confidence intervals. Meta-analysis results showing that individual study data are presented in Supplementary Materials: eFigures 4 and 5 for part A and part B, respectively. 6MWD: 6-minute walking distance, No.: number, CI: confidence interval, TQP: Tai Chi and Qigong Practice, NA: not available, TC\&QG: Tai Chi and Qigong, RM: random-effects model, MCID: minimum clinically important difference.

significant when compared to general exercise controls, it is clinically significant as it exceeds the minimum important clinical difference of 30 meters [51].

The improvements in LVEF and BNP provide further objective evidence (with a moderate level of certainty) for TQPs' benefit in patients with CHF. The pooled effect of LVEF and BNP is consistent with previous research [46]. The possible mechanisms for the improvement observed in LVEF and BNP in trials of TQP therapy have yet to be established. TQPs, as a moderate-intensity exercise, could improve the parasympathetic nervous degree, inhibit sympathetic activity, and increase coronary collateral circulation, cardiac stroke volume, and cardiac output. In this way, it could reduce BNP and increase LVEF [56].

It is also worth noting that no adverse events were reported, and patient dropout in the TQP groups was low, with most withdrawals being due to hospitalization or $\mathrm{CHF}$ exacerbation. The included RCTs showed that participants had good adherence to TQP programs. These results indicate that TQPs are safe and engender good patient compliance.

\section{Limitations}

There are several limitations to this review:
Firstly, the interpretation of our results may be challenged by the heterogeneity observed. Sensitivity analyses by TQP styles or durations revealed some sources of heterogeneity but were unable to account for all of the variations. For example, practitioner expertise, heart failure etiopathogenesis, NYHA classification, and patients with different cultural backgrounds were each revealed to be potential sources of heterogeneity. As many individuals or combined factors may have influenced heterogeneity, this study did not succeed in identifying the reasons for this.

Secondly, the descriptions of the 33 RCTs regarding the randomization method, allocation concealment, and blinding evaluation were neither detailed nor comprehensive. Therefore, the included studies might exhibit moderate selection, implementation, and measurement biases. Because of the intervention itself, participants could identify they were in the experimental group. Trials with inadequate blinding are likely to exaggerate treatment effects, especially with regard to subjective results and with participants with knowledge of Chinese traditional culture.

Thirdly, training characteristics regarding training intensity are rarely described adequately enough to 


\begin{tabular}{|c|c|c|c|c|c|c|c|c|}
\hline & Subgroups & $\begin{array}{l}\text { No. of } \\
\text { studies }\end{array}$ & $\begin{array}{c}\text { No. of } \\
\text { patients }\end{array}$ & $\begin{array}{l}\text { Statistical } \\
\text { method }\end{array}$ & Mean difference $(95 \% \mathrm{CI})$ & $I^{2}$ & Favor control & Favor TQPs \\
\hline \multicolumn{9}{|c|}{ A. TQPs vs no exercise } \\
\hline Total & All & 4 & 245 & FM & $1.24(0.91$ to 1.57$)$ & 0 & & t) \\
\hline \multirow[t]{2}{*}{ TQP types } & -Tai Chi & 3 & 190 & FM & $1.14(068$ to 1.61$)$ & 0 & & + \\
\hline & -Qigong & 1 & 55 & FM & $1.35(0.88$ to 1.82$)$ & $\mathrm{NA}$ & & - \\
\hline \multirow[t]{3}{*}{ Duration } & -4 weeks & 1 & 55 & FM & $1.35(0.88$ to 1.82$)$ & NA & & $\frac{1}{T-}$ \\
\hline & -12 weeks & 2 & 130 & FM & $0.21(-1.36$ to 1.78$)$ & 0 & & \\
\hline & -24 weeks & 1 & 60 & FM & $1.23(0.74$ to 1.72$)$ & NA & & - \\
\hline \multicolumn{9}{|c|}{ B. TOPs vs general exercise } \\
\hline Total & All & 2 & 125 & FM & $0.14(-0.43$ to 0.70$)$ & 0 & & \\
\hline \multirow[t]{2}{*}{ TQP types } & -Tai Chi & 1 & 16 & $\mathrm{FM}$ & $2.20(-2.80$ to 7.20$)$ & NA & & - \\
\hline & -Qiugong & 1 & 109 & FM & $0.11(-0.46$ to 0.68$)$ & NA & & \\
\hline \multirow[t]{3}{*}{ Duration } & -12 weeks & 2 & 125 & FM & $0.14(-0.43$ to 0.70$)$ & 0 & & \\
\hline & & & & & & & & MCID: $\Delta>1 \mathrm{~mL} / \mathrm{kg} / \mathrm{min}$ \\
\hline & & & & & & & 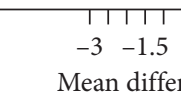 & 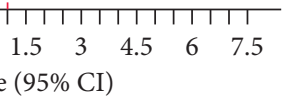 \\
\hline
\end{tabular}

Figure 4: Meta-analysis results for peak VO2 $(\mathrm{mL} / \mathrm{kg} / \mathrm{min})$ including overall pooled effects and subgroup effects. The pooled effect from all included studies and each subgroup is shown in red diamond and blue square, respectively. Both are along with their 95\% confidence intervals. Meta-analysis results showing that individual study data are presented in Supplementary Materials: eFigures 6 and 7 for part A and part B, respectively. VO2: oxygen consumption, No.: number, CI: confidence interval, TQP: Tai Chi and Qigong Practice, NA: not available, RM: random-effects model, FM: fixed-effects model, MCID: minimum clinically important difference.

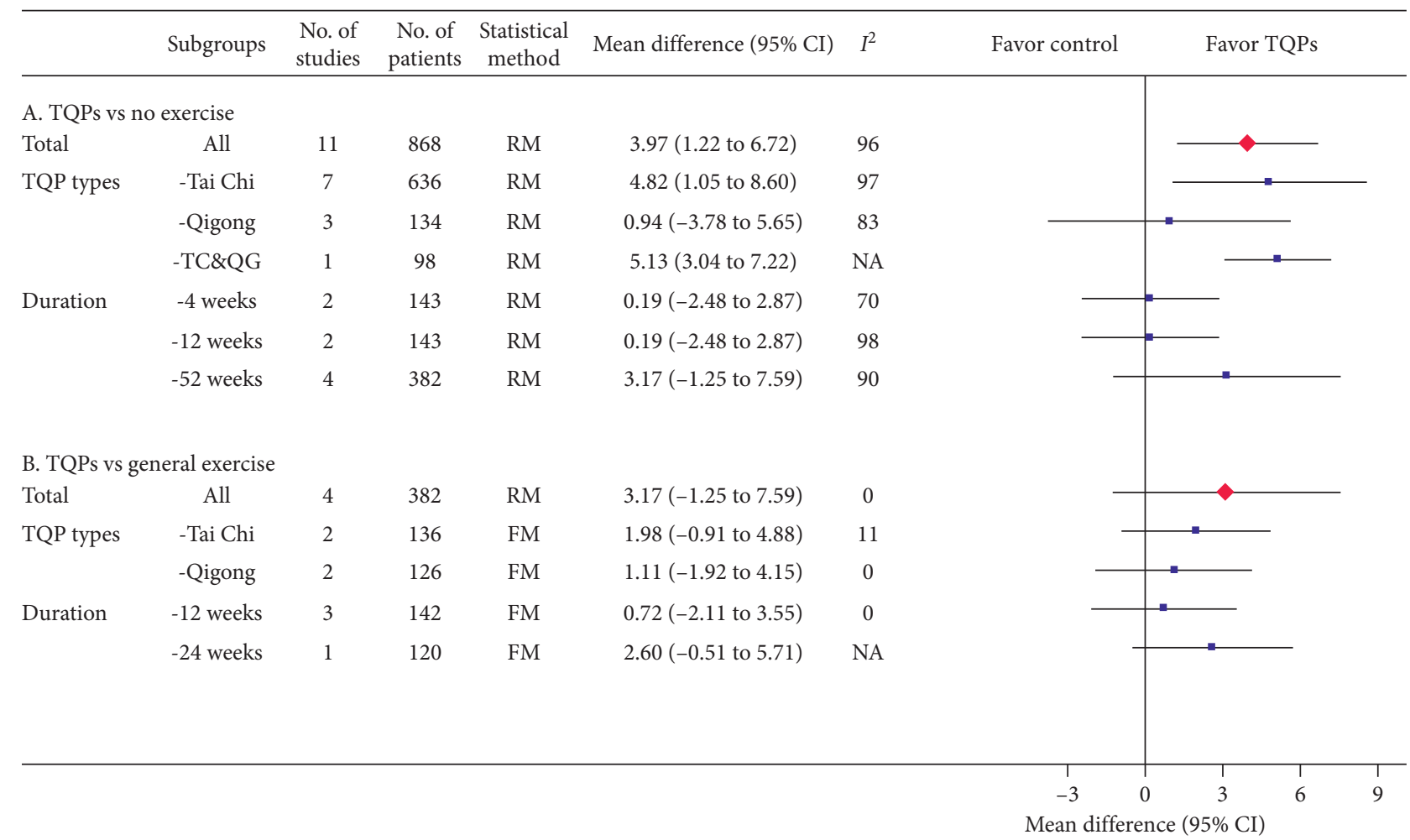

Figure 5: Meta-analysis results for LVEF (\%) including overall pooled effects and subgroup effects. The pooled effect from all included studies and each subgroup is shown in red diamond and blue square, respectively. Both are along with their $95 \%$ confidence intervals. Meta-analysis results showing that individual study data are presented in Supplementary Materials: eFigures 8 and 9 for part A and part B, respectively. LVEF: left ventricular ejection fraction, No.: number, CI: confidence interval, TQP: Tai Chi and Qigong Practice, NA: not available, TC\&QG: Tai Chi and Qigong, RM: random-effects model, FM: fixed-effects model, MCID: minimum clinically important difference. 


\begin{tabular}{|c|c|c|c|c|c|c|c|c|c|c|}
\hline & Subgroups & $\begin{array}{l}\text { No. of } \\
\text { studies }\end{array}$ & $\begin{array}{l}\text { No. of } \\
\text { patients }\end{array}$ & $\begin{array}{c}\text { Statistical } \\
\text { method }\end{array}$ & Mean difference (95\% CI) & $I^{2}$ & & Favor TQPs & Favor control & \\
\hline \multicolumn{11}{|c|}{ A. TQPs vs no exercise } \\
\hline Total & All & 8 & 691 & $\mathrm{RM}$ & $-76.12(-134.61$ to -17.62$)$ & 95 & & $\longrightarrow$ & & \\
\hline \multirow[t]{2}{*}{ TQP types } & -Tai Chi & 4 & 279 & $\mathrm{RM}$ & -73.44 ( -196.86 to 49.98$)$ & 96 & & 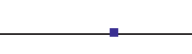 & & \\
\hline & -Qigong & 4 & 412 & $\mathrm{RM}$ & $-52.10(-75.21$ to -28.99$)$ & 42 & & $\longrightarrow$ & & \\
\hline \multirow[t]{4}{*}{ Duration } & -4 weeks & 1 & 88 & $\mathrm{RM}$ & $-0.18(-40.90$ to 40.54$)$ & NA & & - & 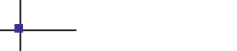 & \\
\hline & -12 weeks & 3 & 230 & $\mathrm{RM}$ & $-38.81(-52.17$ to -25.45$)$ & 0 & & $\rightarrow$ & & \\
\hline & -24 weeks & 1 & 61 & $\mathrm{RM}$ & $-181.00(-201.29$ to -160.71$)$ & NA & $\rightarrow$ & & & \\
\hline & -52 weeks & 3 & 312 & $\mathrm{RM}$ & $-72.87(-116.33$ to -29.41$)$ & 18 & & $\longrightarrow$ & & \\
\hline \multicolumn{11}{|c|}{ B. TQPs vs general exercise } \\
\hline Total & All & 2 & 136 & $\mathrm{RM}$ & $61.29(-52.22$ to 174.79$)$ & 77 & & & - & \\
\hline TQP types & -Tai Chi & 2 & 136 & RM & $61.29(-52.22$ to 174.79$)$ & 77 & & & & \\
\hline \multirow[t]{3}{*}{ Duration } & -12 weeks & 1 & 16 & $\mathrm{RM}$ & $-9.00(-114.68$ to 96.68$)$ & NA & & 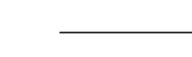 & - & \\
\hline & -24 weeks & 1 & 120 & $\mathrm{RM}$ & $109.00(78.41$ to 139.59$)$ & NA & & & $\longrightarrow$ & \\
\hline & & & & & & & $\begin{array}{c}1 \\
-200\end{array}$ & $\begin{array}{l}1 \\
-100\end{array}$ & 100 & $\begin{array}{c}1 \\
200\end{array}$ \\
\hline
\end{tabular}

Figure 6: Meta-analysis results for BNP (pg/mL) including overall pooled effects and subgroup effects. The pooled effect from all included studies and each subgroup is shown in red diamond and blue square, respectively. Both are along with their $95 \%$ confidence intervals. Metaanalysis results showing that individual study data are presented in Supplementary Materials: eFigures 10 and 11 for part A and part B, respectively. BNP: B-type natriuretic peptide, No.: number, CI: confidence interval, TQP: Tai Chi and Qigong Practice, NA: not available, RM: random-effects model, MCID: minimum clinically important difference.

TABLe 2: Meta-analysis results of clinical outcomes with more than one study.

\begin{tabular}{|c|c|c|c|c|c|c|}
\hline \multirow{2}{*}{ Outcomes measurements } & \multirow{2}{*}{ No. of RCTs } & \multirow{2}{*}{ No. of patients } & \multirow{2}{*}{$\begin{array}{l}\text { Statistical } \\
\text { method }\end{array}$} & \multicolumn{2}{|l|}{ Effect sizes } & \multirow{2}{*}{$\begin{array}{l}\text { Heterogeneity } \\
\quad\left(I^{2}\right)(\%)\end{array}$} \\
\hline & & & & MD $(95 \% \mathrm{CI})$ & $p$ value & \\
\hline \multicolumn{7}{|l|}{$\begin{array}{l}\text { A. (Tai Chi and Qigong Practice } \\
\text { plus RM) vs. (RM) }\end{array}$} \\
\hline NT-proBNP, pg/mL & 6 & 350 & MD, REM & $-232.05(-578.87$ to 114.78$)$ & 0.19 & 97 \\
\hline $\mathrm{VO}_{2 \mathrm{AT}}, \mathrm{ml} / \mathrm{kg} / \mathrm{min}$ & 2 & 115 & MD, REM & $1.43(0.59$ to 2.28$)$ & 0.0009 & 93 \\
\hline LVEDd, $\mathrm{mm}$ & 4 & 397 & MD, REM & $-3.00(-5.09$ to -0.91$)$ & 0.005 & 63 \\
\hline Depression & 6 & 462 & SMD, REM & $-0.64(-1.03$ to -0.25$)$ & 0.001 & 76 \\
\hline Anxiety & 2 & 163 & SMD, FEM & $-1.00(-2.41$ to 0.41$)$ & 0.17 & 94 \\
\hline Mood state & 2 & 160 & SMD, REM & $-0.08(-1.47$ to 1.31$)$ & 0.91 & 94 \\
\hline Fatigue & 2 & 125 & SMD, REM & $0.01(-1.07$ to 1.09$)$ & 0.98 & 88 \\
\hline Norepinephrine & 2 & 130 & $\mathrm{MD}, \mathrm{FEM}$ & $0.51(-0.71$ to 1.72$)$ & 0.41 & 0 \\
\hline SF-36-bodily pain & 2 & 159 & MD, REM & $5.84(0.62$ to 11.06$)$ & 0.03 & 80 \\
\hline SF-36-mental health & 2 & 159 & MD, REM & $6.55(1.78$ to 11.32$)$ & 0.007 & 58 \\
\hline SF-36-physical function & 2 & 159 & $\mathrm{MD}, \mathrm{FEM}$ & $6.73(4.05$ to 9.42$)$ & $<0.00001$ & 20 \\
\hline SF-36-role emotional & 2 & 159 & MD, FEM & $5.60(2.78$ to 8.43$)$ & $<0.0001$ & 48 \\
\hline SF-36-role physical & 2 & 159 & MD, REM & $9.87(0.52$ to 19.22$)$ & 0.04 & 92 \\
\hline SF-36-social function & 2 & 159 & MD, FEM & $6.78(4.09$ to 9.47$)$ & $<0.00001$ & 0 \\
\hline SF-36-vitality & 2 & 159 & $\mathrm{MD}, \mathrm{REM}$ & $8.28(0.77$ to 15.79$)$ & 0.03 & 91 \\
\hline Hospitalizations per capita & 3 & 322 & MD, FEM & $-0.82(-0.95$ to -0.69$)$ & $<0.00001$ & $I^{2}=0$ \\
\hline Hospitalization cost per capita & 3 & 322 & $\mathrm{MD}, \mathrm{FEM}$ & $-1.60(-1.85$ to -1.36$)$ & $<0.00001$ & $I^{2}=0$ \\
\hline \multicolumn{7}{|c|}{$\begin{array}{l}\text { B.(Tai Chi and Qigong Practice } \\
\text { plus RM) vs. (general exercises plus RM) }\end{array}$} \\
\hline NT-proBNP, pg/mL & 3 & 186 & MD, FEM & $-12.01(-23.65$ to -0.37$)$ & 0.04 & 0 \\
\hline $\mathrm{SBP}, \mathrm{mmHg}$ & 2 & 76 & $\mathrm{MD}, \mathrm{FEM}$ & $-9.60(-22.02$ to 2.83$)$ & 0.13 & 0 \\
\hline $\mathrm{DBP}, \mathrm{mmHg}$ & 2 & 76 & $\mathrm{MD}, \mathrm{FEM}$ & $0.82(-5.27$ to 6.92$)$ & 0.79 & 0 \\
\hline Depression & 2 & 63 & SMD, FEM & $-0.28(-0.77$ to 0.22$)$ & 0.28 & 0 \\
\hline
\end{tabular}

RCT: randomized controlled trial, CI: confidence interval, RM: routine management, NT-proBNP: $N$-terminal B-type natriuretic peptide, $\mathrm{VO}_{2 \mathrm{AT}}$ : oxygen consumption at anaerobic threshold, MD: mean difference, SMD: standardized mean difference, REM: random-effects model, FEM: fixed-effects model, LVEDd: left ventricular end-diastolic end-systolic diameters, SBP: systolic blood pressure, DBP: diastolic blood pressure. 
evaluate TQP's dose-response effects, i.e., to render the studies replicable, or to interpret the findings' validity and translate the interventions into practice. This might be due to the fact that there is still no standardized method to measure TQPs' intensity. As exercise intensity is reported to be the most critical component for improving cardiorespiratory fitness [57], future research on TQPs should provide descriptions of intensity, in combination with frequency, duration, and adherence.

Lastly, the majority of this review's sample is from China, but people from different cultures may experience variegated responses to TQPs. Moreover, rehabilitation-related trials have been investigating the efficacy of exercises in selective populations, such as women, the elderly, ethnic minorities, HFpEF, and high-risk patients. Future research might also identify subgroups that benefit the most/least from TQPs.

\section{Conclusions}

The findings of this systematic review and meta-analysis suggest that based on moderate-level evidence, adding TQPs into RMs was associated with statistically significant improvements to the quality of life and exercise capacity in CHF patients. In addition, evidence from RCTs indicated (with a low level of certainty) that TQPs may have similar rehabilitation effects as general aerobic exercises. Considering the lack of special equipment requirements, low costs, and multiple physical benefits, TQPs may represent a promising rehabilitation therapy as an adjunct to routine pharmacotherapies or as an alternative to conventional exercises. Incorporation into cardiac rehabilitation programs for patients with CHF should be considered, especially in home-based settings.

\section{Abbreviations}

6MWD: 6-minute walking distance

BNP: B-type natriuretic peptide

CHF: Chronic heart failure

CI: $\quad$ Confidence intervals

EBCR: Exercise-based cardiac rehabilitation

IQR: Interquartile range

LVEF: Left ventricular ejection fraction

MD: $\quad$ Mean difference

MLHFQ: Minnesota Living with Heart Failure

Questionnaire

NYHA: New York Heart Association

RCT: Randomized controlled trial

RM: $\quad$ Routine management

SD: $\quad$ Standard deviation

SMD: $\quad$ Standardized mean difference

TQP: $\quad$ Tai Chi and Qigong Practice

$\mathrm{VO}_{2 \text { peak }}$ : Peak oxygen consumption.

\section{Data Availability}

Data are available upon request to the corresponding author.

\section{Conflicts of Interest}

The authors declare that there are no conflicts of interest regarding the publication of this paper.

\section{Acknowledgments}

The authors thank Shaojun Liao from the Guangzhou University of Traditional Chinese Medicine for assistance with data extraction. This work was supported by the General Research Fund of Traditional Chinese Medicine Science and Technology from the Guangdong Provincial Hospital of Chinese Medicine (YN2018ML02) and the Clinical Research Fund of the Traditional Chinese Medicine Science and Technology (Project 1010) from Guangdong Provincial Hospital of Chinese Medicine (YN10101910). This study was part of a Ph.D. thesis supported by the China Scholarships Council (201608440264).

\section{Supplementary Materials}

eTable 1: sensitivity analysis of the effect size of Peak VO2, 6MWD, MLHFQ, LVEF, and BNP. eFigure 1: flow chart of study identification and selection. eFigure 2: MLHFQ total scores, TQPs plus RMs versus RMs (supplements to Figure 2(a)). eFigure 3: MLHFQ total scores, TQPs plus RMs versus general exercises plus RMs (supplements to Figure 2(b)). eFigure 4: 6MWD (meters), TQPs plus RMs versus RMs (supplements to Figure 3(a)). eFigure 5: 6MWD (meters), TQPs plus RMs versus general exercises plus RMs (supplements to Figure 3(b)). eFigure 6: Peak VO2 (mL/kg/ min), TQPs plus RMs versus RMs (supplements to Figure 4(a)). eFigure 7: Peak VO2 (mL/kg/min), TQPs plus RMs versus general exercises plus RMs (supplements to Figure 4(b)). eFigure 8: LVEF (\%), TQPs plus RMs versus RMs (supplements to Figure 5(a)). eFigure 9: LVEF (\%), TQPs plus RMs versus general exercises plus RMs (supplements to Figure 5(b)). eFigure 10: BNP (pg/mL), TQPs plus RMs versus RMs (supplements to Figure 6(a)). eFigure 11: BNP (pg/mL), TQPs plus RMs versus general exercises plus RMs (supplements to Figure 6(b)). eFigure 12: risk-of-bias summary: eFigure 13: risk-of-bias graph. eFigure 14: publication bias. (Supplementary Materials)

\section{References}

[1] G. Savarese and L. H. Lund, "Global public health burden of heart failure," Cardiac Failure Review, vol. 3, no. 1, pp. 7-11, 2017.

[2] K. Turk-Adawi, N. Sarrafzadegan, and S. L. Grace, "Global availability of cardiac rehabilitation," Nature Reviews Cardiology, vol. 11, no. 10, pp. 586-596, 2014.

[3] V. M. Conraads, C. Deaton, E. Piotrowicz et al., "Adherence of heart failure patients to exercise: barriers and possible solutions," European Journal of Heart Failure, vol. 14, no. 5, pp. 451-458, 2012.

[4] J. L. Fleg, L. S. Cooper, B. A. Borlaug et al., "Exercise training as therapy for heart failure," Circulation: Heart Failure, vol. 8, no. 1, pp. 209-220, 2015.

[5] J. Qu and X. Wang, Traditional Chinese Exercises, Cambridge Scholars Publishing, Newcastle-upon-Tyne, UK, 2015. 
[6] C. L.-W. Chan, C.-W. Wang, R. T.-H. Ho et al., "A systematic review of the effectiveness of qigong exercise in cardiac rehabilitation," The American Journal of Chinese Medicine, vol. 40, no. 2, pp. 255-267, 2012.

[7] X. Q. Wang, P. Yan-Ling, C. Pei-Jie et al., "Traditional Chinese exercise for cardiovascular diseases: systematic review and meta-analysis of randomized controlled trials," Journal of American Heart Association, vol. 5, no. 3, Article ID e002562, 2016.

[8] D. Moher, A. Liberati, J. Tetzlaff, and D. G. Altman, "Preferred reporting items for systematic reviews and meta-analyses: the PRISMA statement,” BMJ, vol. 339, Article ID b2535, 2009.

[9] J. P. Higgins and S. Green, Cochrane Handbook for Systematic Reviews of Interventions, The Cochrane Collaboration, London, UK, 2018, http://www.handbook.cochrane.org.

[10] I. Boutron, D. Moher, D. G. Altman, K. F. Schulz, and P. Ravaud, "Extending the CONSORT statement to randomized trials of nonpharmacologic treatment: explanation and elaboration," Annals of Internal Medicine, vol. 148, no. 4, pp. 295-309, 2008.

[11] T. C. Hoffmann, P. P. Glasziou, I. Boutron et al., "Better reporting of interventions: template for intervention description and replication (TIDieR) checklist and guide," BMJ, vol. 348, Article ID g1687, 2014.

[12] L. Zheng, C. Zhang, X. K. Wu, A. Gao, H. M. Li, and X. Wang, "Influence of healthcare qigong-6-character formulas (Liuzijue) on heart function in patients with chronic heart failure," Chinese Journal of Evidence-Based Cardiovascular Medicine, vol. 6, pp. 659-662, 2017.

[13] L. S. Redwine, W. Kathleen, A. P. Meredith et al., "A randomized study examining the effects of mild-to-moderate group exercises on cardiovascular, physical, and psychological well-being in patients with heart failure," Journal of Cardiopulmonary Rehabilitation Prevention, vol. 39, no. 6, pp. 403-408, 2019.

[14] T. Yu, "Effects of Taijiquan rehabilitation program at different training times on cardiac function in patients with chronic heart failure," Chinese Journal of Integrative Medicine on Cardio-/Cerebrovascuiar Disease, vol. 17, no. 12, pp. 1772-1775, 2019, in Chinese.

[15] D. E. Barrow, A. Bedford, G. Ives, L. O’Toole, and K. S. Channer, "An evaluation of the effects of Tai Chi Chuan and Chi Kung training in patients with symptomatic heart failure: a randomised controlled pilot study," Postgraduate Medical Journal, vol. 83, no. 985, pp. 717-721, 2007.

[16] G. Caminiti, V. Maurizio, M. Giuseppe et al., "Tai chi enhances the effects of endurance training in the rehabilitation of elderly patients with chronic heart failure," Rehabilitation Research Practice, vol. 2011, Article ID 761958, 6 pages, 2011.

[17] D. M. Chen, Y. Wen-Chung, H. Huei-Fong, T. Jen-Chen, W. Hsiao-Ying, and C. Ai-Fu, "The effects of Baduanjin exercise on fatigue and quality of life in patients with heart failure: a randomized controlled trial," European Journal Cardiovascular Nursing, vol. 17, no. 5, pp. 456-466, 2017.

[18] W. Feng, "Effect of Taijiquan on cardiopulmonary function in patients with chronic heart failure," Master thesis in Chinese, Liaoning University of Traditional Chinese Medicine, Shenyang, China, 2017.

[19] C. Huang, "Study on the rehabilitation of cardiac function in patients with chronic heart failure (NYHA class III) with coronary heart disease," Master thesis in Chinese, Fujian University of Traditional Chinese Medicine, Fuzhou, China, 2014.
[20] C. Li, Y. Yu, and Y. Fang, "Effect of exercise tolerance in patients with chronic heart failure exercise training," Unrsing Practice and Research, vol. 12, no. 12, pp. 36-37, 2015.

[21] R. Li, "Observation on the improvement of cardiac function and quality of life in elderly patients with chronic heart failure by Ba Duan Jin," Massage and Rehabilitation Medicine, vol. 8, no. 24, pp. 23-25, 2017, in Chinese.

[22] X. Pan, "Effects of Taijiquan on cardiac function and quality of life in patients with chronic heart failure," Chinese Journal of Physical Medicine and Rehabilitation, vol. 38, no. 1, pp. 51-53, 2016.

[23] L. S. Redwine, M. Tsuang, A. Rusiewicz et al., "A pilot study exploring the effects of a 12-week T'ai Chi intervention on somatic symptoms of depression in patients with heart failure," The Journal of Alternative and Complementary Medicine, vol. 18, no. 8, pp. 744-748, 2012.

[24] L. Sang, "Effect of Taiji rehabilitation exercise on cardiac function and quality of life in elderly patients with coronary heart disease and chronic heart failure," Chinese Journal of Gerontology, vol. 35, no. 14, pp. 3957-3958, 2015.

[25] N. Wang, "The research on chronic heart failure by Taijiquan with drugs," Master thesis in Chinese, Nanjing University of Traditional Chinese Medicine, Nanjing, China, 2011.

[26] X. Xiong and D. Xu, "Therapeutic effect of Baduanjin on patients with coronary heart disease and chronic heart failure," China Modern Medicine Journal, vol. 18, no. 5, pp. 55-56, 2016, in Chinese.

[27] X. Xiong, "Efect of BaDuanJin on cognitive function of patiens with coronary heart disease complicated with chronic heart failure," Clinical Medical \& Engineering, vol. 24, no. 12, pp. 1723-1724, 2017.

[28] X. Yan, "Effects of Liuzi and Ba Duan Jin training on exercise tolerance in patients with chronic heart failure," Chinese Traditional Medicine Modern Distance Education, vol. 14, no. 16, pp. 126-128, 2016, in Chinese.

[29] X. Yang, "Effect of aerobics training on exercise tolerance in patients with chronic heart failure," Chinese Journal of Nursing, vol. 50, no. 2, pp. 193-197, 2015, in Chinese.

[30] C. Yao, Li Fu, and Y. Ma, "Effect of Taijiquan exercise on rehabilitation of patients with chronic heart failure," Journal of Cardiovascular Rehabilitation Medicine, vol. 19, no. 4, pp. 364-367, 2010, in Chinese.

[31] G. Y. Yeh, M. J. Wood, B. H. Lorell et al., "Effects of tai chi mind-body movement therapy on functional status and exercise capacity in patients with chronic heart failure: a randomized controlled trial," The American Journal of Medicine, vol. 117, no. 8, pp. 541-548, 2004.

[32] G. Y. Yeh, E. P. McCarthy, P. M. Wayne et al., "Tai chi exercise in patients with chronic heart failure: a randomized clinical trial," Archives Internal Medicine, vol. 171, no. 8, pp. 750-757, 2011.

[33] G. Y. Yeh, M. J. Wood, P. M. Wayne et al., "Tai chi in patients with heart failure with preserved ejection fraction," Congestive Heart Failure, vol. 19, no. 2, pp. 77-84, 2013.

[34] J. Yu, "A study on the effect of TCM aerobics on exercise endurance in patients with chronic heart failure," Chinese Medicine Modern Distance Education of China, vol. 13, no. 21, pp. 12-14, 2015, in Chinese.

[35] L. Yuan, "Effect of Taijiquan on depression, sleep quality and quality of life in elderly patients with chronic congestive heart failure and depression," Guangxi Medicine, vol. 35, no. 11, pp. 1547-1550, 2016, in Chinese.

[36] Z. Shi, "Clinical study of Baduanjin on rehabilitation treatment of patients with chronic heart failure," Master thesis in 
Chinese, Liaoning University of Traditional Chinese Medicine, Shenyang, China, 2018.

[37] M. Yu, A randomized controlled study on the application of Baduanjin in patients with coronary heart disease and chronic heart failure, $\mathrm{PhD}$ thesis in Chinese, Beijing University of Traditional Chinese Medicine, Beijing, China, 2018.

[38] H. Liu, "Clinical effect of Taijiquan on chronic heart failure with coronary heart disease," Master thesis in Chinese, Guangzhou University of Traditional Chinese Medicine, Guangdong, China, 2017.

[39] X. Li, "A randomized parallel controlled study of Baduanjin combined with western medicine for chronic ejection fraction retention and chronic heart failure," Journal of Practical Traditional Chinese Internal Medicine, vol. 33, no. 3, pp. 56-59, 2019.

[40] D. Yu and Z. Jiang, "Effects of Taijiquan and Baduanjin on exercise tolerance and quality of life in patients with chronic heart failure," Journal of New Chinese Medicine, vol. 51, no. 3, pp. 274-277, 2019, in Chinese.

[41] X. Deng, D. Yu, and X. Zhang, "Effects of Taijiquan exercise on cardiac function and psychology of patients with cardiac insufficiency after acute myocardial infarction," Journal of North Sichuan Medical College, vol. 33, no. 4, pp. 545-547, 2018, in Chinese.

[42] H. Lu, "Effects of seated Baduanjin on quality of life in patients with heart function grade III IV stable heart failure," Cardiovascular Disease Journal Of Integrated Traditional Chinese and Western Medicine, vol. 7, no. 8, pp. 1-2+4, 2019.

[43] L. Hägglund, K. Boman, and M. Brännström, "A mixed methods study of Tai Chi exercise for patients with chronic heart failure aged 70 years and older," Nursing Open, vol. 5, no. 2, pp. 176-185, 2018.

[44] R. Zheng, "Chan-Chuang Qigong improves exercise capacity, depression, and quality of life in patients with heart failure," Hu Li Za Zhi, vol. 65, no. 5, pp. 34-44, 2018.

[45] D. T. Wade, "Outcome measures for clinical rehabilitation trials: impairment, function, quality of life, or value?" American Journal of Physical Medicine Rehabilitation, vol. 82, no. 10, pp. S26-S31, 2003.

[46] Q. Gu, S.-J. Wu, Y. Zheng et al., "Tai chi exercise for patients with chronic heart failure: a meta-analysis of randomized controlled trials," American Journal of Physical Medicine \& Rehabilitation, vol. 96, no. 10, pp. 706-716, 2017.

[47] X. Ren, L. Yanda, Y. Xinyu et al., "The effects of tai chi training in patients with heart failure: a systematic review and metaanalysis," Front Physiology, vol. 8, p. 989, 2017.

[48] L. Pan, J. Yan, Y. Guo, and J. Yan, "Effects of Tai Chi training on exercise capacity and quality of life in patients with chronic heart failure: a meta-analysis," European Journal of Heart Failure, vol. 15, no. 3, pp. 316-323, 2013.

[49] I. L. Piña, C. S. Apstein, G. J. Balady et al., "Exercise and heart failure: a statement from the American Heart Association Committee on exercise, rehabilitation, and prevention," Circulation, vol. 107, no. 8, pp. 1210-1225, 2003.

[50] L. Bernardi, G. Spadacini, J. Bellwon, R. Hajric, H. Roskamm, and A. W. Frey, "Effect of breathing rate on oxygen saturation and exercise performance in chronic heart failure," The Lancet, vol. 351, no. 9112, pp. 1308-1311, 1998.

[51] M. J. Shoemaker, A. B. Curtis, E. Vangsnes, and M. G. Dickinson, "Clinically meaningful change estimates for the six-minute walk test and daily activity in individuals with chronic heart failure," Cardiopulmonary Physical Therapy Journal, vol. 24, no. 3, pp. 21-29, 2013.
[52] R. Malhotra, K. Bakken, E. D’Elia, and G. D. Lewis, "Cardiopulmonary exercise testing in heart failure," JACC: Heart Failure, vol. 4, no. 8, pp. 607-616, 2016.

[53] L. Long, I. R. Mordi, C. Bridges et al., "Exercise-based cardiac rehabilitation for adults with heart failure," The Cochrane Database of Systematic Reviews, vol. 1, Article ID Cd003331, 2019.

[54] H. M. Imran, B. Muhammad, E. Sebhat et al., "Home-based cardiac rehabilitation alone and hybrid with center-based cardiac rehabilitation in heart failure: a systematic review and meta-analysis," Journal of American Heart Association, vol. 8, no. 16, Article ID e012779, 2019.

[55] Y. M. Chen and Y. Li, "Safety and efficacy of exercise training in elderly heart failure patients: a systematic review and metaanalysis," International Journal of Clinical Practice, vol. 67, no. 11, pp. 1192-1198, 2013.

[56] X.-G. Sun, "Rehabilitation practice patterns for patients with heart failure: the Asian perspective," Heart Failure Clinics, vol. 11, no. 1, pp. 95-104, 2015.

[57] ACSM and American College of Sports Medicine, ACSM's Guidelines for Exercise Testing and Prescription, Wolters Kluwer/Lippincott Williams \& Wilkins, Philadelphia, PA, USA, 10th edition, 2017. 